\title{
Molecular detection of Leishmania infantum, filariae and Wolbachia spp. in dogs from southern Portugal
}

\author{
Carla Maia ${ }^{1,2^{*}}$ D, Laura Altet ${ }^{3}$, Lorena Serrano ${ }^{3}$, José Manuel Cristóvão ${ }^{1}$, Maria Dolores Tabar ${ }^{4}$, Olga Francino ${ }^{3,5}$,
} Luís Cardoso ${ }^{6}$, Lenea Campino ${ }^{1,7}$ and Xavier Roura ${ }^{8}$

\begin{abstract}
Background: Leishmaniosis caused by the protozoan Leishmania infantum and dirofilariosis caused by the nematodes Dirofilaria immitis or Dirofilaria repens are vector-borne zoonoses widely present in the Mediterranean basin. In addition, some studies reported that the endosymbiont Wolbachia spp. play a role in the biology and pathogenesis of filarial parasites. The aim of this work was to evaluate the frequency of mono- and co-infections by L. infantum, filariae and Wolbachia spp. and their association with clinical signs in dogs from the south of Portugal. Leishmanial, filarial and Wolbachia spp. DNA were evaluated by specific real-time polymerase chain reaction (qPCR) assays in blood samples from 230 dogs.
\end{abstract}

Findings: One hundred and thirty-nine (60.4\%) dogs were qPCR-positive for L. infantum and 26 (11.3\%) for filariae (24 for D. immitis only, one D. immitis and for Acanthocheilonema dracunculoides and another one for Acanthocheilonema reconditum only). Wolbachia spp. DNA was amplified from 16 (64.0\%) out of the 25 D. immitis-positive dogs. Nineteen (8.3\%) dogs were co-infected with L. infantum and D. immitis, including the one (0.4\%) A. drancunculoides-positive animal. In dogs without clinical signs consistent with leishmaniosis and/or dirofilariosis, L. infantum prevalence was $69 \%$, whereas in those dogs with at least one clinical manifestation compatible with any of the two parasitoses prevalence was $42.7 \%$. Leishmania prevalence was significantly higher in apparently healthy mongrels (77.2\%) and pets (76.9 \%) than in defined-breed dogs (including crosses; $58.8 \%$ ) and in dogs with an aptitude other than pet (i.e. farm, guard, hunting, shepherd or stray), respectively, whereas in those dogs with at least one clinical sign, the detection of L. infantum DNA was higher in males (53.3 \%) and in those dogs not receiving insect repellents (52.8 \%).

Conclusions: The molecular detection of canine vector-borne disease (CVBD) agents, some of which are zoonotic, reinforces the need to implement efficient prophylactic measures, such as insect repellents and macrocyclic lactones (including compliance to administration), in the geographical areas where these agents are distributed, with the view to prevent infection and disease among mammalian hosts including humans.

Keywords: Dogs, Dirofilaria immitis, Leishmania infantum, Portugal, Wolbachia spp

\section{Findings}

Canine vector-borne diseases (CVBD) constitute a diversified group of illnesses, which are caused by a multitude of pathogens transmitted by arthropod vectors [1]. In addition to their veterinary importance, dogs play a

\footnotetext{
* Correspondence: carlamaia@ihmt.unl.pt

${ }^{1}$ Global Health and Tropical Medicine, Medical Parasitology Unit, Instituto de Higiene e Medicina Tropical, Universidade Nova de Lisboa, Lisbon, Portugal

${ }^{2}$ Faculty of Veterinary Medicine, Universidade Lusófona de Humanidades e

Tecnologias, Lisbon, Portugal

Full list of author information is available at the end of the article
}

central role in the transmission cycles of some vectorborne agents by acting as reservoirs and sentinels of human infections, thus making the control of CVBD desirable under the One Health umbrella.

Leishmaniosis caused by the protozoan Leishmania infantum and heartworm disease and subcutaneous filariosis respectively caused by the nematodes Dirofilaria immitis and Dirofilaria repens are three vector-borne zoonoses widely present in the Mediterranean basin, with transmission of the first one by phlebotomine sand flies of 
the genus Phlebotomus and of the last two by mosquitoes mainly from the genera Culex, Aedes and Anopheles [2, 3]. Other less known filarial worms endemic in Europe such as Acanthocheilonema dracunculoides and Acanthocheilonema reconditum are transmitted mainly by ticks or by fleas and lice, respectively [4].

Endosymbiont bacteria Wolbachia spp. (order Rickettsiales) have been found in several filarial species, such as D. immitis and D. repens, but not in Achanthocheilonema spp. [5]. Wolbachia organisms seem to play an essential role in the biology of filarial parasites and in the pathogenesis of infections due to these nematodes, potentially increasing the severity of clinical signs $[6,7]$.

Canine leishmaniosis is a systemic chronic condition whose clinical manifestations often include lymphadenomegaly, cutaneous alterations, weight loss, ocular signs, epistaxis, onychogryphosis and lameness [8]. Canine dirofilariosis is associated with a dry chronic cough, exercise intolerance, dyspnoea, weakness, weight loss, epistaxis, cyanosis and even congestive heart failure [7]. Both parasitoses are endemic in the south of Portugal. Leishmania seroprevalence in dogs has ranged from $3.8 \%$ in randomly screened apparently healthy animals from the Algarve [9] to $40.6 \%$ in dogs from the same region that were clinically suspect of leishmaniosis [10]. The detection of $D$. immitis antigen has ranged from $2.4 \%$ in apparently healthy dogs from Lisbon to $17.1 \%$ in dogs from the Algarve with clinical signs compatible with a CVBD [9]. Wolbachia spp. DNA has also been detected in D. immitis infected dogs from the centre and south of Portugal [11, 12], and $A$. reconditum and $A$. drancunculoides infections have been reported in animals from the same areas [13, 14]. Furthermore, D. repens microfilariae were recently detected in one dog from the Algarve region, the southernmost region of continental Portugal [15].

Vector-borne pathogen co-infections may lead to an increased severity of clinical signs as previously shown in dogs from southeastern Spain with leishmaniosis and/ or filariosis [16]. On the other hand, a protective role of Wolbachia limiting the severity of leishmaniosis was also observed in dogs co-infected with $L$. infantum and $D$. immitis [16]. As the presence of co-infections may lead to a non-characteristic clinical outcome which will further complicate the diagnosis, treatment and prognosis, together with the zoonotic potential of L. infantum and Dirofilaria spp., the aim of this work was to evaluate the prevalence of mono- and co-infections by L. infantum, filariae and Wolbachia and their association with clinical signs consistent with leishmaniosis or dirofilariosis in dogs from the south of Portugal.

From May 2011 to February 2014, a total of 230 dogs from veterinary medical centres and animal shelters in southern Portugal randomly selected (i.e. out of any dog present to the veterinary clinic or any dog living in the shelter) were studied (Table 1$)$. The dogs were from the districts of Setúbal ( $n=68$, including 13 dogs from the contiguous districts of Évora and Beja) and Faro $(n=$ 162). Domestic dogs were included after informed consent was obtained from the owners. In the case of stray dogs, a written consent for enrolment was obtained from their legal detainer, i.e. the person in charge of the rescue association.

Whenever available, data on gender, breed, age, life style, living conditions, prophylactic use of sand fly and/or mosquito repellents and of macrocyclic lactones, and clinical status, i.e. absence (Table 1) or presence (Table 2) of at least one sign compatible with leishmaniosis or dirofilariosis) were registered for each dog. Clinical signs comprised anorexia, cough, dermatological manifestations, dyspnoea, epistaxis, exercise intolerance, gastrointestinal alterations, lameness, lethargy, lymphadenopathy, muscular atrophy, onychogryphosis, ocular manifestations, pale mucous and weight loss.

This study was ethically approved by the boards of the IHMT-UNL and of the Faculty of Veterinary Medicine (ULHT) as complying with the Portuguese legislation for the protection of animals (Decree-Law no. 113/2013).

Whole blood samples were collected by cephalic or jugular venipuncture and spotted onto filter paper. Samples were dried at room temperature and kept at $4{ }^{\circ} \mathrm{C}$ until DNA extraction by a commercial kit (Kit Citogene ${ }^{\oplus}$, Citomed). Four discs of filter paper $(4 \mathrm{~mm}$ in diameter each) were incubated with lysis buffer $(150 \mu \mathrm{l})$ and $1.5 \mu \mathrm{l}$ of proteinase $\mathrm{K}(20 \mathrm{mg} / \mathrm{ml})$. Further DNA extraction followed the kit manufacturer's instructions.

All the samples were submitted for real-time PCR (qPCR) for $L$. infantum, filariae (D. immitis, D. repens, A. dracunculoides and $A$. reconditum) and Wolbachia spp. Leishmania quantitative PCR was performed as previously described by Francino et al. [17]. qPCRs for Spirurida (Filariidae, Onchocercidade and Thelaziidae) and Wolbachia were carried out in a final volume of $20 \mu \mathrm{L}$ using SYBR Select (Life Technologies), $0.1 \mu \mathrm{M}$ of each primer for Wolbachia species (described by Tabar et al. [16]) and $0.5 \mu \mathrm{M}$ of each primer for Spirurida (5'-CGTAATTTTARTWCTTCTTTTTATGAT RCTA-3'; 5' -CCAAAYAAACGWTCCTTATCAGTYAA$\left.3^{\prime}\right)$ and $4 \mu \mathrm{L}$ of DNA (10-100 ng). The thermal cycling profile was $50{ }^{\circ} \mathrm{C}$ for $2 \mathrm{~min}$ and $95{ }^{\circ} \mathrm{C}$ for $10 \mathrm{~min}$ followed by 40 cycles at $95{ }^{\circ} \mathrm{C}$ for $15 \mathrm{~s}$ and $60{ }^{\circ} \mathrm{C}$ for $1 \mathrm{~min}$. The eukaryotic 18S RNA Pre-Developed TaqMan Assay Reagent (Life Technologies) was used as internal reference for dog genomic DNA amplification to ensure proper qPCR amplification of each sample and that negative results corresponded to true negative samples rather than to a problem with DNA loading, sample degradation or PCR inhibition. Positive qPCR controls were obtained from clinical samples that had been previously amplified and sequenced to confirm the pathogen and water was used as a 
Table 1 Molecular prevalence of L. infantum, filariae and Wolbachia among dogs with no clinical signs compatible with leishmaniosis or dirofilariosis $(n=155)$

\begin{tabular}{|c|c|c|c|c|c|}
\hline \multirow[t]{3}{*}{ Variable/category } & \multirow{3}{*}{$\begin{array}{l}\text { No. (\%) of } \\
\text { tested dogs }\end{array}$} & \multicolumn{4}{|c|}{ Percentage $(n)$ of positive dogs } \\
\hline & & $\overline{P C R}$ & PCR & PCR & $\geq 1$ agent \\
\hline & & L. infantum & filariae & Wolbachia spp. & \\
\hline Gender & 154 & & & & \\
\hline Female & $84(54.5)$ & $70.2(59)$ & $13.1(11)$ & $6.0(5)$ & $72.6(61)$ \\
\hline Male & $70(45.5)$ & $68.6(48)$ & $11.4(8)$ & $7.1(5)$ & $70.0(49)$ \\
\hline Age (months) & 143 & & & & \\
\hline$[1-11]$ & $23(16.1)$ & $60.9(14)$ & $4.3(1)$ & $0.0(0)$ & $60.9(14)$ \\
\hline$[12-83]$ & $82(57.3)$ & $74.4(61)$ & $13.4(11)$ & $6.1(5)$ & $76.8(63)$ \\
\hline [84-192] & $38(26.6)$ & $60.5(23)$ & $15.8(6)$ & $13.2(5)$ & $63.2(24)$ \\
\hline Breed & 147 & & & & \\
\hline Defined (incl. crosses) & $68(46.3)$ & $58.8(40)^{\mathrm{a}}$ & $11.8(8)$ & $5.9(4)$ & $63.2(43)$ \\
\hline Mongrel & $79(53.7)$ & $77.2(61)^{\mathrm{a}}$ & $12.7(10)$ & $7.6(6)$ & $77.2(61)$ \\
\hline Aptitude & 155 & & & & \\
\hline Pet & $108(69.7)$ & $76.9(83)^{b}$ & $12.0(13)$ & $5.6(6)$ & $79.6(86)^{c}$ \\
\hline Other $^{1}$ & $47(30.3)$ & $51.1(24)^{b}$ & $12.8(6)$ & $8.5(4)$ & $51.1(24)^{c}$ \\
\hline Housing & 145 & & & & \\
\hline Indoors and mixed & $75(51.7)$ & $61.3(46)$ & $9.3(7)$ & $5.3(4)$ & $64.0(48)$ \\
\hline Outdoors & $70(48.3)$ & $75.7(53)$ & $17.1(12)$ & $8.6(6)$ & $77.1(54)$ \\
\hline Repellent insecticides ${ }^{2}$ & 87 & & & & \\
\hline Yes & $30(34.5)$ & $54.4(31)$ & $8.8(5)$ & $3.5(2)$ & $57.9(33)$ \\
\hline No & $57(65.5)$ & $43.3(13)$ & $10.0(3)$ & $6.7(2)$ & $46.7(14)$ \\
\hline Macrocyclic lactones ${ }^{3}$ & 54 & & & & \\
\hline Yes & $13(24.1)$ & $38.5(5)$ & $0.0(0)$ & $0.0(0)$ & $38.5(5)$ \\
\hline No & $41(75.9)$ & $31.7(13)$ & $17.1(7)$ & $14.6(6)$ & $36.6(15)$ \\
\hline Total & 155 (100) & $69.0(107)$ & $12.3\left(19^{*}\right)$ & $6.5(10)$ & $71.0(110)$ \\
\hline
\end{tabular}

${ }^{1}$ Farm, guard, hunting, shepherd or stray; ${ }^{2}$ Deltamethrin, flumethrin and/or permethrin; ${ }^{3}$ Ivermectin, mimemycin oxime or moxydectin; ${ }^{\mathrm{a}} P=0.026 ;{ }^{\mathrm{b}} P=0.003 ;{ }^{\mathrm{c}} P=$ 0.001 ; ${ }^{*}$ Comprising 18 cases of $D$. immitis infection and one case of $A$. dracunculoides and $D$. immitis co-infection

PCR-negative control. The L. infantum load in blood was quantified according to Delta $\mathrm{Ct}$, namely: low DeltaCt $>15$; medium DeltaCt 5-15; high DeltaCt $<5$ and very high DeltaCt negative. Spirurida qPCR positive samples were sequenced by the Big-Dye Terminator Cycle Sequencing Ready reaction Kit (Life Technologies) using the same primers. Sequences obtained were compared with GenBank database (www.ncbi.nlm.nih.gov/BLAST).

Percentages of positivity for L. infantum, filariae and Wolbachia spp. regarding the independent variables and categories were compared by the Chi-square or Fisher's exact tests. A $P$ value $\leq 0.05$ was considered as statistically significant. Exact binomial $95 \%$ confidence intervals (CI) were defined for the proportions. Analyses were performed with SPSS $^{\bullet} 21$ software for Windows and with StatLib.

This study was carried out with a considerable number of dogs $(n=230)$, which were mainly owned and tame ones. On the contrary, previous investigations on canine filarial infections in southern Portugal had either been carried out exclusively with shelter dogs $[13,14]$ or with not so many $(n=157)$ domestic dogs [12].

One hundred and thirty-nine dogs were qPCR-positive to L. infantum. Out of the 97 dogs tested from November to April (Leishmania non-transmission period) 72 (74.2\%; CI: 64.3-82.6\%) were positive, whereas from May to October (transmission period) L. infantum DNA was detected in 67 (50.4\%; CI: 41.6-59.2\%) of 133 canine blood samples $(P<0.001)$. The significantly higher $(P<0.001)$ Leishmania prevalence obtained in the present study, i.e. $69.0 \%$ (CI: 61.1-76.2 \%) in dogs with no clinical signs of leishmaniosis and/or dirofilariosis (Table 1) and $42.7 \%$ (CI: 31.3-54.6\%) in dogs with compatible clinical signs (Table 2) compared with the $1.1 \%$ recently obtained in dogs from the south of the country by conventional PCR [18], could be related to the higher sensitivity of the qPCR, which is able to detect less than one parasite per millilitre of blood [17]. This most recent figure of $69.0 \%$ is more in 
Table 2 Molecular prevalence of L. infantum, filariae and Wolbachia in dogs with clinical signs compatible with leishmaniosis and/or dirofilariosis $(n=75)$

\begin{tabular}{|c|c|c|c|c|c|}
\hline \multirow[t]{3}{*}{ Variable/category } & \multirow{3}{*}{$\begin{array}{l}\text { No. (\%) of } \\
\text { tested dogs }\end{array}$} & \multicolumn{4}{|c|}{ Percentage (n) of positive dogs } \\
\hline & & $\overline{P C R}$ & PCR & $P C R$ & $\geq 1$ \\
\hline & & L. infantum & filariae & Wolbachia spp. & \\
\hline Gender & 75 & & & & \\
\hline Female & $32(42.7)$ & $28.1(9)^{a}$ & $12.5(4)$ & $9.4(3)$ & $34.4(11)$ \\
\hline Male & $43(57.3)$ & $53.5(23)^{a}$ & $7.0(3)$ & $7.0(3)$ & $55.8(24)$ \\
\hline Age (months) & 67 & & & & \\
\hline$[1-11]$ & $1(1.5)$ & $0.0(0)$ & $0.0(0)$ & $0.0(0)$ & $0.0(0)$ \\
\hline [12-83] & $33(49.3)$ & $39.4(13)$ & $12.1(4)$ & $12.1(4)$ & $45.5(15)$ \\
\hline [84-192] & $33(49.3)$ & $42.4(14)$ & $9.1(3)$ & $6.1(2)$ & $45.5(15)$ \\
\hline Breed & 73 & & & & \\
\hline Defined (incl. crosses) & $40(54.8)$ & $40.0(16)$ & $7.5(3)$ & $7.5(3)$ & $42.5(17)$ \\
\hline Mongrel & $33(45.2)$ & $48.5(16)$ & $12.1(4)$ & $9.1(3)$ & $54.5(18)$ \\
\hline Aptitude & 75 & & & & \\
\hline Pet & $37(49.3)$ & $40.5(15)$ & $16.2(6)$ & $13.5(5)$ & $48.6(18)$ \\
\hline Other ${ }^{1}$ & $38(50.7)$ & $44.7(17)$ & $2.6(1)$ & $2.6(1)$ & $44.7(17)$ \\
\hline Housing & 41 & & & & \\
\hline Indoors and mixed & $18(43.9)$ & $27.8(5)$ & $5.6(1)$ & $5.6(1)$ & $33.3(6)$ \\
\hline Outdoors & $23(56.1)$ & $47.8(11)$ & $17.4(4)$ & $13.0(3)$ & $52.2(12)$ \\
\hline Repellent insecticides ${ }^{2}$ & 55 & & & & \\
\hline Yes & $19(34.5)$ & $21.1(4)^{b}$ & $10.5(2)$ & $5.3(1)$ & $26.3(5)$ \\
\hline No & $36(65.5)$ & $52.8(19)^{b}$ & $5.6(2)$ & $5.6(2)$ & $55.6(20)$ \\
\hline Macrocyclic lactones ${ }^{3}$ & 37 & & & & \\
\hline Yes & $9(24.3)$ & $0.0(0)$ & $0.0(0)$ & $0.0(0)$ & $0.0(0)$ \\
\hline No & $28(75.7)$ & $25.0(7)$ & $10.7(3)$ & $7.1(2)$ & $32.1(9)$ \\
\hline Total & $75(100)$ & $42.7(32)$ & $9.3\left(7^{*}\right)$ & $8.0(6)$ & $46.7(35)$ \\
\hline
\end{tabular}

${ }^{1}$ Farm, guard, hunting or stray; ${ }^{2}$ Deltamethrin or permethrin; ${ }^{3}$ Ivermectin, milbemycin oxime or moxydectin; ${ }^{\mathrm{a}} P=0.050$; ${ }^{\mathrm{b}} P=0.048$; ${ }^{*}$ Comprising six dogs positive for $D$. immitis and another one for $A$. reconditum

accordance with previously reported molecular prevalence values clearly above $50 \%$ [16]. Nevertheless, it cannot be ruled out that positive blood results were due to transient contamination during the transmission season and may thus represent just exposure to the parasite rather than an established infection.

A negative association was found between Leishmania positivity and clinical signs compatible with leishmaniosis and/or dirofilariosis (69.0\% [Table 1] versus $42.7 \%$ [Table 2]; $P<0.001)$. The fact that more dogs considered clinically healthy harboured Leishmania parasites in their circulation might be due to a fact most of the enrolled dogs were randomly selected from those attending veterinarian clinics and, therefore, probably receiving more medical care, better nutrition, and for that reason were less prone to develop disease. The prevalence of $L$. infantum (76.9 \%; CI: 67.7-84.4 \%; $P=0.003$ ) and that of infection with at least one pathogen ( $\geq 1$ agent; $79.6 \%$; CI: $70.8-86.8 \% ; P=0.001)$ in dogs without compatible clinical signs was significantly higher in pets in comparison with dogs with other aptitudes (Table 1). The present results, therefore, disagree with those that revealed a higher prevalence of infection in guard dogs as compared to pets in the Madrid region, Spain [19]. These latter findings might be somewhat due to a different lifestyle of pets (i.e. urban vs. rural) in distinct geographic areas. The higher prevalence of $L$. infantum DNA in clinically healthy mongrel dogs in comparison with those belonging to defined breeds or their crosses $(P=0.026)$ might be related with a certain level of resistance to disease development and progression by the mixed-breed infected dogs [20].

In those dogs showing clinical signs compatible with leishmaniosis and/or dirofilariosis the detection of $L$. infantum DNA in the blood was significantly higher $(P$ =0.050; Table 2) in males (53.5\%; CI: 37.6-68.8\%). Gender predisposition to the infection has been considered by some authors as a non-determinant factor for 
leishmaniosis [19, 20], while others have reported a higher prevalence in male dogs $[21,22]$. On the other hand, the use of long-acting topical insecticides on dogs has been shown to be an effective measure in reducing the prevalence of Leishmania infection [23]. Therefore, it is not entirely surprising that the prevalence of Leishmania was higher in those dogs not protected against insects $(P=0.048 ;$ Table 2$)$. In fact, and although the number of effective molecules for prophylaxis against leishmaniosis has increased in the last few years, our results clearly reinforce the idea that there is still a long way to go regarding the prevention and control of Leishmania infection.

Filarial infections were detected in 26 (11.3 \%; CI: 7.5$16.1 \%)$ blood samples: 24 (10.4 \%; CI: 6.8-15.1 \%) dogs harboured D. immitis only, one (0.4 \%; CI: $0.01-2.4 \%)$ $\operatorname{dog} A$. reconditum only and another one (0.4\%; CI: $0.01-$ $2.4 \%) A$. drancunculoides in co-infection with D. immitis. This corroborates the previous reports on the circulation of these nematodes in the south and central regions of Portugal $[9,12-14]$. D. immitis prevalence in the present study (10.9 \%; CI: 7.2-15.6 \%) was lower (although nonsignificantly; $P=0.111$ ) than the overall prevalence of $15.1 \%(105 / 696)$ recently obtained in three coastal areas of central and southern-central Portugal [14]. Differences can be related to the dynamics of infection over time, which is probably linked to the distribution and abundance of the vectors and to the surveyed canine population. In fact, in the work performed by Alho et al. [14] all the screened animals were sheltered dogs that lived outdoors and had probably not received any prophylactic measure for heartworm infection, thus being more exposed to vectors and to the agents they might transmit. On the other hand, in the present study most of the tested dogs were pets living partially indoors and receiving veterinary health care. Interestingly, and although the difference was not statistically significant $(P=0.562), D$. immitis DNA was not detected in any of the dogs receiving macrocyclic lactones, suggesting the importance of using this prophylactic measure in areas where dirofilariosis is endemic. As the compliance to the use of ectoparasiticides was not evaluated, it cannot be ruled out whether the reason for no differences between the use of insecticides/repellents and the presence of heartworm infection was due to irregular administration of insecticides.

The detection of Wolbachia DNA in 16 (64.0\%; CI: 42.5-82.0\%) dogs positive to $D$. immitis was higher than the $52.6 \%(20 / 38 ; P=0.372)$ and $30.6 \%(15 / 49 ; P=$ $0.006)$ recently obtained in Portuguese [11] and Spanish [16] dogs, respectively. As reported by previous publications, only $D$. immitis and $D$. repens have ever been found infected with Wolbachia, but not all the specimens of these filarial parasites transport the endosymbiont bacteria. Taken together, these findings suggest that in endemic areas a combined PCR for Wolbachia and Dirofilaria should be performed for the diagnosis of heartworm, in order to avoid a high percentage of falsenegative Dirofilaria results due to the lack of testing for the endosymbiont.

Wolbachia DNA was detected in 5 (31.3 \%; CI: $11.0-$ $58.7 \%)$ dogs solely infected with $D$. immitis and in 11 (68.8 \%; CI: 41.3-89.0\%; $P=0.034$ ) co-infected dogs (11 with L. infantum-D. immitis and one with $A$. dracunculoides-D.immitis), reinforcing that this endosymbiont does not seem to be present in Achanthocheilonema spp. alone [5]. On the other hand, our results contrast with a previous study carried out in dogs with leishmaniosis and/or dirofilariosis from Spain where the prevalence of the endosymbiont was significantly lower in microfilariaemic dogs co-infected with L. infantum [16]. Interestingly, in the present study only three out of the 11 Wolbachia, D. immitis and L. infantum coinfected dogs presented clinical signs compatible with leishmaniosis and/or dirofilariosis, which might have been due to the stimulation of a Th1 type protectiveimmune response triggered by the nematode and the endosymbiont bacterium [16, 24].

In the present study, 19 (8.3 \%; CI: 5.0-12.6\%) dogs were co-infected with $L$. infantum and $D$. immitis. It should be stressed that the occurrence of co-infections with these vector-borne agents, which are relatively common in dogs living in geographic areas where competent vectors for the different pathogens co-exist, might induce more severe and atypical clinical outcomes that will further complicate diagnosis, treatment and prognosis $[9,16,25]$. However, this hypothesis has not been corroborated in the present study and the reason might be the random screening of the canine population, with most dogs showing no clinical signs, even when they were positive for one or more of the studied agents.

The on-going and recurrent detection of zoonotic vector-borne agents in dogs with or without clinical signs reinforces the importance of increasing the veterinary community, owners and public health authorities' awareness regarding the risk of infection. It also highlights the need to apply efficient prophylactic measures, such as insect repellents and macrocyclic lactones (including compliance to administration), not only by the local owners but also by the tourists from non-endemic countries vacationing with their dogs in endemic areas, in order to prevent infection and disease among mammalian hosts including humans. Nevertheless, as environmental changes, global warming, and growing world trade and animal transportation, including the increased mobility of dogs across borders, have an impact on the geographic distribution, abundance and vectorial capacity of arthropods, the expansion of L. infantum and filariae to new locations should not be neglected. The 
present results further stress the need for sustained development of multi-pathogen detection methods in regions endemic and even non-endemic for CVBD.

\section{Competing interests}

The authors declare that they have no competing interests.

\section{Authors' contributions}

CM planned, designed and supervised the study, and wrote the manuscript; $L A$ and LS performed GPCR assays; JMC performed DNA extraction; LuC performed data analysis and revised the manuscript; MDT, OF and LeC reviewed the manuscript; XR planned the study and reviewed the manuscript. All authors read and approved the final manuscript.

\section{Acknowledgements}

This work was partially supported by Funds to GHTM (UID/Multi/04413/ 2013). The authors thank the cooperation of veterinarians, auxiliary staff, dog owners and shelters that contributed with collection of samples. The work of C. Maia and L. Cardoso was done under the frame of EurNegVec COST Action TD1303.

Publication of this paper has been sponsored by Bayer Animal Health in the framework of the 11th CVBD World Forum Symposium.

\section{Author details}

${ }^{1}$ Global Health and Tropical Medicine, Medical Parasitology Unit, Instituto de Higiene e Medicina Tropical, Universidade Nova de Lisboa, Lisbon, Portugal. ${ }^{2}$ Faculty of Veterinary Medicine, Universidade Lusófona de Humanidades e Tecnologias, Lisbon, Portugal. ${ }^{3}$ Vetgenomics, Parc de Recerca Universitat Autònoma de Barcelona, Bellaterra, Barcelona, Spain. ${ }^{4}$ Hospital Veterinario San Vicente, San Vicente del Raspeig, Alicante, Spain. ${ }^{5}$ SVGM, Departamento de Ciencia Animal y de los Alimentos, Facultad de Veterinària, Universitat Autònoma de Barcelona, Bellaterra, Barcelona, Spain. ${ }^{6}$ Department of Veterinary Sciences, School of Agrarian and Veterinary Sciences, University of Trás-os-Montes e Alto Douro, Vila Real, Portugal. ${ }^{7}$ Department of Biomedical Sciences and Medicine, Universidade do Algarve, Faro, Portugal. ${ }^{8}$ Hospital Clínic Veterinari, Universitat Autònoma de Barcelona, Bellaterra, Barcelona, Spain.

Received: 17 January 2016 Accepted: 15 March 2016

Published online: 10 May 2016

\section{References}

1. Dantas-Torres F, Otranto D. Best Practices for preventing vector-borne diseases in dogs and humans. Trends Parasitol. 2015. doi:10.1016/.jpt.2015.09.004.

2. Ferreira CA, de Pinho MV, Novo MT, Calado MM, Gonçalves LA, Belo SM, et al. First molecular identification of mosquito vectors of Dirofilaria immitis in continental Portugal. Parasit Vectors. 2015;8:139.

3. Maia C, Parreira R, Cristóvão JM, Freitas FB, Afonso MO, Campino L. Molecular detection of Leishmania DNA and identification of blood meals in wild caught phlebotomine sand flies (Diptera: Psychodidae) from southern Portugal. Parasit Vectors. 2015;8:173.

4. Otranto D, Dantas-Torres F, Brianti E, Traversa D, Petrić D, Genchi C, et al. Vectorborne helminths of dogs and humans in Europe. Parasit Vectors. 2013;6:16.

5. Bandi C, Tress AJ, Bratting NW. Wolbachia in filarial nematodes: evolutionary aspects and implications for the pathogenesis and treatment of filarial diseases. Vet Parasitol. 2001;98:215-38

6. Kramer L, Grandi G, Leoni M, Passeri B, McCall J, Genchi C, et al. Wolbachia and its influence on the pathology and immunology of Dirofilaria immitis infection. Vet Parasitol. 2008:158:191-5.

7. McCall JW, Genchi C, Kramer LH, Guerrero J, Venco L. Heartworm disease in animals and humans. Adv Parasitol. 2008;66:193-285.

8. Campino L, Maia C. The role of reservoirs: canine leishmaniasis. In: PonteSucre A, Padron-Nieves M, Diaz E, editors. Drug resistance in Leishmania parasites - consequences, molecular mechanism and possible treatments. Vienna: Springer Verlag; 2013. p. 45-64.

9. Cardoso L, Mendão C, Madeira de Carvalho L. Prevalence of Dirofilaria immitis, Ehrlichia canis, Borrelia burgdorferi sensu lato, Anaplasma spp. and Leishmania infantum in apparently healthy and CVBD-suspect dogs in Portugal - a national serological study. Parasit Vectors. 2012;5:62.

10. Maia C, Dionísio L, Afonso MO, Neto L, Cristóvão JM Campino L. Leishmania infection and host-blood feeding preferences of phlebotomine sandflies and canine leishmaniasis in an endemic European area, the Algarve Region in Portugal. Mem Inst Oswaldo Cruz. 2013:108:481-7.

11. Landum M, Ferreira CC, Calado M, Alho AM, Maurício IL, Meireles JS, et al. Detection of Wolbachia in Dirofilaria infected dogs in Portugal. Vet Parasitol. 2014;204:407-10.

12. Maia C, Coimbra M, Ramos C, Cristóvão JM, Cardoso L, Campino L. Serological investigation of Leishmania infantum. Dirofilaria immitis and Angiostrongylus vasorum in dogs from southern Portugal. Parasit Vectors. 2015:8:152.

13. Menn B, Lorentz S, Naucke TJ. Imported and travelling dogs as carriers of canine vector-borne pathogens in Germany. Parasit Vectors. 2010;3:34.

14. Alho AM, Landum M, Ferreira C, Meireles J, Gonçalves L, de Carvalho LM, et al. Prevalence and seasonal variations of canine dirofilariosis in Portugal. Vet Parasitol. 2014;206:99-105.

15. Maia C, Lorentz S, Cardoso L, Otranto D, Naucke TJ. Detection of Dirofilaria repens microfilariae in a dog from Portugal. Parasitol Res. 2015;115:441-3.

16. Tabar MD, Altet L, Martínez V, Roura X. Wolbachia, filariae and Leishmania coinfection in dogs from a Mediterranean area. J Small Anim Pract. 2013;54:174-8.

17. Francino O, Altet L, Sánchez-Robert E, Rodriguez A, Solano-Gallego L, Alberola J, et al. Advantages of real-time PCR assay for diagnosis and monitoring of canine leishmaniosis. Vet Parasitol. 2006;137:214-21.

18. Maia C, Almeida B, Coimbra M, Fernandes MC, Cristóvão JM, Ramos C, et al. Bacterial and protozoal agents of canine vector-borne diseases in the blood of domestic and stray dogs from southern Portugal. Parasit Vectors. 2015;8:138.

19. Galvez R, Miró G, Descalzo MA, Nieto J, Dado D, Martín O, et al. Emerging trends in the seroprevalence of canine leishmaniasis in the Madrid region (central Spain). Vet Parasitol. 2010;169:327-34.

20. Cortes S, Vaz Y, Neves R, Maia C, Cardoso L, Campino L. Risk factors for canine leishmaniasis in an endemic Mediterranean region. Vet Parasitol. 2012:189:189-96.

21. Zivicnjak T, Martinković F, Marinculić A, Mrljak V, Kucer N, Matijatko V, et al. A seroepidemiologic survey of canine visceral leishmaniosis among apparently healthy dogs in Croatia. Vet Parasitol. 2005;131:35-43.

22. Miranda S, Roura X, Picado A, Ferrer L, Ramis A. Characterization of sex, age, and breed for a population of canine leishmaniosis diseased dogs. Res Vet Sci. 2008:85:35-8.

23. Courtenay O, Kovacic V, Gomes P, Garcez L, Quinnell R. A long-lasting topical deltamethrin treatment to protect dogs against visceral leishmaniasis. Med Vet Entomol. 2009;23:245-56.

24. Oleaga A, Pérez-Sánchez R, Pagés E, Marcos-Atxutegi C, Simón F. Identification of immunoreactive proteins from the dog heartworm (Dirofilaria immitis) differentially recognized by the sera from dogs with patent or occult infections. Mol Biochem Parasitol. 2009:166:134-41.

25. De Tommasi AS, Otranto D, Dantas-Torres F, Capelli G, Breitschwerdt EB, de Caprariis D. Are vector-borne pathogen co-infections complicating the clinical presentation in dogs? Parasit Vectors. 2013;6:97.

\section{Submit your next manuscript to BioMed Central} and we will help you at every step:

- We accept pre-submission inquiries

- Our selector tool helps you to find the most relevant journal

- We provide round the clock customer support

- Convenient online submission

- Thorough peer review

- Inclusion in PubMed and all major indexing services

- Maximum visibility for your research

Submit your manuscript at www.biomedcentral.com/submit
C) Biomed Central 